\title{
Identification of hub genes and key pathways associated with the progression of gynecological cancer
}

\author{
XI $\mathrm{ZHANG}^{1}$ and $\mathrm{YUDONG}_{\mathrm{WANG}}^{2}$ \\ ${ }^{1}$ Department of Gynecology, Changning Maternity and Infant Health Hospital, Shanghai 200051; ${ }^{2}$ Department of Gynecology, \\ International Peace Maternity and Child Health Hospital, Shanghai Jiao Tong University, Shanghai 200030, P.R. China
}

Received October 2, 2018; Accepted June 5, 2019

DOI: $10.3892 / \mathrm{ol} .2019 .11004$

\begin{abstract}
Gynecological cancer is the leading cause of cancer mortality in women. However, the mechanisms underlying gynecological cancer progression have remained largely unclear. In the present study, 799 dysregulated genes were identified in ovarian serous cystadenocarcinoma (OV), 488 dysregulated genes in cervical squamous cell carcinoma and endocervical adenocarcinoma (CESC), and 621 dysregulated genes in uterine corpus endometrial carcinoma (UCEC). Bioinformatics analysis revealed that mRNA splicing and cell proliferation-associated biological processes served important roles in $\mathrm{OV}$ progression. Metabolism-associated biological processes played important roles in CESC progression, and protein phosphorylation and small GTPase-mediated signal transduction served important roles in UCEC progression. The present study also constructed OV, CESC and UCEC progression-associated protein-protein interaction networks to reveal the associations among these genes. Furthermore, Kaplan-Meier curve analysis showed that progression-related genes were associated with the duration of overall survival. Finally, NARS2 and TPT1 in OV, SMYD2, EGLN1, TNFRSF10D, FUT11, SYTL3, MMP8 and EREG in CESC, and SLC5A1, TXN, KDM4B, TXNDC11, HSDL2, COX16, MGAT4A, DAGLA, ELOVL7, THRB and PCOLCE2 in UCEC were identified as hub genes in cancer progression. Therefore, this study may assist in the identification of novel mechanisms underlying cancer progression and new biomarkers for gynecological cancer prognosis and therapy.
\end{abstract}

Correspondence to: Dr Yudong Wang, Department of Gynecology, International Peace Maternity and Child Health Hospital, Shanghai Jiao Tong University, 773 WuYi Road, Shanghai 200030, P.R. China E-mail: wangyudong201x@sina.com

Key words: ovarian serous cystadenocarcinoma, uterine corpus endometrial carcinoma, cervical squamous cell carcinoma and endocervical adenocarcinoma, protein-protein interaction analysis, biomarker

\section{Introduction}

In the past decades, gynecological cancer has been the leading cause of cancer mortality in women globally (1). The major types of gynecological cancer include ovarian serous cystadenocarcinoma (OV), uterine corpus endometrial carcinoma (UCEC), and cervical squamous cell carcinoma and endocervical adenocarcinoma (CESC) (2). Despite the various therapeutic methods that have been developed, including surgical, hormone therapeutic and chemotherapeutic treatments, the 5-year survival rate of patients with gynecological cancer has remained poor $(3,4)$. For example, the 5-year overall survival (OS) of patients with late-stage OV is $<20 \%$ (3). The advanced-stage CESC 5-year OS rate has remained as low as $30 \%$ (4). Of note, the mechanisms underlying gynecological cancer require further investigation. Exploration the potential regulators involved in gynecological cancer progression is urgently needed to identify novel biomarkers of cancer prognosis and targets for treatment.

Next-generation sequencing (NGS) is an important tool in the generation of new cancer therapies and diagnostic methods $(5,6)$. The Cancer Genome Atlas (TCGA) database, including $>30$ types of human cancer, is the most widely used NGS database and has played a crucial role in the discovery of cancer-associated genes and mutations $(7,8)$. For example, Sanchez-Vega et al (9) analyzed oncogenic signaling pathways across 33 human cancer types using TCGA datasets. In gynecological cancer, a series of key regulators were also identified by using similar strategies. For instance, Berger et al (10) identified a series of mutated genes and somatic copy-number alterations in gynecological cancer by comprehensively analyzing TCGA datasets. Song et al (11) constructed an aberrant long noncoding RNA-microRNA-mRNA network in CESC using TCGA datasets. Comprehensive analysis of TCGA datasets provides novel insights into the mechanisms involved in tumor progression to allow for the identification of new biomarkers for human cancer, including gynecological cancer.

In order to obtain a comprehensive assessment of the molecular mechanisms underlying gynecological cancer progression, a bioinformatics analysis of OV, CESC and USEC datasets from TCGA was conducted to identify hub genes and key pathways in the present study. In addition, the prognostic value of these key genes in gynecological cancer was also evaluated. 


\section{Materials and methods}

TCGA dataset analysis. In the present study, TCGA CESC, $\mathrm{OV}$ and UCEC datasets were downloaded from the cBioPortal system (12). Level 3 RNA sequencing version 2 data were downloaded from TCGA (https://www.cbioportal.org/). A total of 233 stage I + II CESC and 68 stage III + IV CESC samples were included in TCGA CESC dataset. A total of 23 stage I + II OV and 282 stage III + IV OV samples were included in TCGA OV dataset. A total of 122 stage I + II UCEC and 54 stage III + IV UCEC samples were included in TCGA UCEC dataset. All specimens were independently assessed by two experienced pathologists according to the 8th edition of the American Joint Committee on Cancer (AJCC) TNM staging system (13). Gene expression with $\mathrm{P}<0.01$ between early-stage (stage I + II) and advanced-stage (stage III + IV) samples was identified to indicate significantly differential expression. Hierarchical cluster analysis was performed, and a hierarchical clustering heat map was generated for the abnormally expressed genes using CLUSTER version 3.0 (14) and the Tree View system (15).

Protein-protein interaction (PPI) networks and module analysis. PPI networks were constructed to reveal the relationships among gynecological cancer progression-associated genes following two steps. Firstly, the combined score between each protein-protein pair was calculated using STRING version 11.0 (http://www.string-db.org/); reliable protein-protein interactions (combined score, $>0.4$ ) were selected for PPI network construction. Secondly, an analysis of the degrees of each node was performed, and the key nodes (node degree $\geq 5$ ) in the PPI network were retained using Cytoscape software (version 3.6.0; http://www.cytoscape.org/).

Gene ontology $(G O)$ and pathway analysis. The Database for Annotation, Visualization and Integrated Discovery (DAVID) version 6.8 system (https://david.ncifcrf.gov/tools.jsp) provides a comprehensive set of functional annotation tools to identify disease-associated biological processes (16). Therefore, DAVID was used to conduct GO analysis $(17,18)$. The top 15 associated 'biological processes' (BPs) are shown. The analysis results of molecular functions and Cellular Component were not shown in this study. BPs with $\mathrm{P}<0.05$ were considered to be significant.

Survival analysis of differentially expressed genes (DEGs). In order to examine whether these DEGS could be the potential biomarkers for the prognosis of gynecological cancer, Kaplan-Meier analysis and log-rank tests were conducted using an online public database, GEPIA (http://gepia.cancer-pku. $\mathrm{cn} /$ index.html). Patients with gynecological cancer were categorized into 2 groups depending on the expression levels in cancer samples; the median expression of candidate genes in all tumor samples was selected as the cut-off point to divide gynecological cancer samples in to high- or low-expression groups. $\mathrm{P}<0.05$ was considered to indicate a statistically significant difference.

Statistical analysis. Differences in gene expression between the individual groups were analyzed using unpaired Student's t-test or Mann-Whitney U-test. PASW Statistics 23.0 software from SPSS Inc. was used. $\mathrm{P}<0.05$ was considered to indicate a statistically significant difference.

\section{Results}

Identification of DEGs in the progression of gynecological cancer. Datasets from TCGA were downloaded to identify DEGs in OV, CESC and UCEC progression. Gene expression with $\mathrm{P}<0.01$ between low-stage (stage I + II) and advanced-stage (stage III + IV) samples was identified to indicate differential expression. A total of 153, 335 and 406 upregulated genes and 646, 153 and 215 downregulated genes were identified in OV, CESC and UCEC progression, respectively. Hierarchical clustering showed DEGs in higher-stage compared with lower-stage OV (Fig. 1A), CESC (Fig. 1B) and UCEC (Fig. 1C) samples.

In order to understand whether common or cancer-specific genes drive the progression of gynecological cancer, the dysregulated genes were compared. As shown in Fig. 1, no common dysregulated genes were observed in OV, CESC and UCEC (Fig. 1C and D). Meanwhile, only 11 upregulated and 21 downregulated genes were found in two types of gynecological cancer (Fig. 1C and D). These results suggested that different genes regulate cancer progression in different types of gynecological cancer.

Bioinformatics analysis of DEGs in gynecological cancer. Next, bioinformatics analysis was performed on the DEGs in gynecological cancer. GO analysis revealed that the DEGs associated with $\mathrm{OV}$ progression were mainly involved in regulating 'mRNA splicing', 'transcription', 'G2/M transition of mitotic cell cycle', 'cellular response to DNA damage stimulus', 'mitophagy', 'protein phosphorylation', 'cell-cell adhesion', 'cell division' and 'DNA repair' (Fig. 2A). DEGs in CESC progression were mainly involved in regulating 'gluconeogenesis', 'response to muramyl dipeptide', 'protein import into nucleus', 'canonical glycolysis', 'circadian regulation of translation', 'apoptotic cell clearance', 'positive Notch signaling pathway', 'glycolytic process', 'protein kinase activity' and 'oxygen homeostasis' (Fig. 2B). The study also indicated that DEGs in UCEC were associated with 'protein phosphorylation', 'small GTPase mediated signal transduction', 'transcription', 'intracellular signal transduction', 'hippo signaling', 'cytoskeleton organization', 'negative regulation of execution phase of apoptosis', 'bicellular tight junction assembly', 'positive regulation of apoptotic process' and 'protein destabilization' (Fig. 2C).

Construction of progression-associated gene-mediated PPI networks in gynecological cancer. As presented in Fig. 3, the OV progression-associated PPI networks included 258 proteins and 1,872 edges (Fig. 3A). The top 10 hub genes with highest degrees involved in OV progression were identified, including EHMT1,EHMT2, BRCA1, PRDM10,CKAP5, SNRNP70, ATR, $M T O R, S E T D 2$ and MIB2. The UCEC progression-associated PPI networks included 99 proteins and 375 edges (Fig. 3B). The top 10 hub genes with the highest degrees involved in UCEC progression were identified, including RHOA, ISG15, LATS2, ACTL8, CDK2, SPTB, TTK, EDN1, FBXO41 and 
A

OV

Stage I+II Stage III+IV
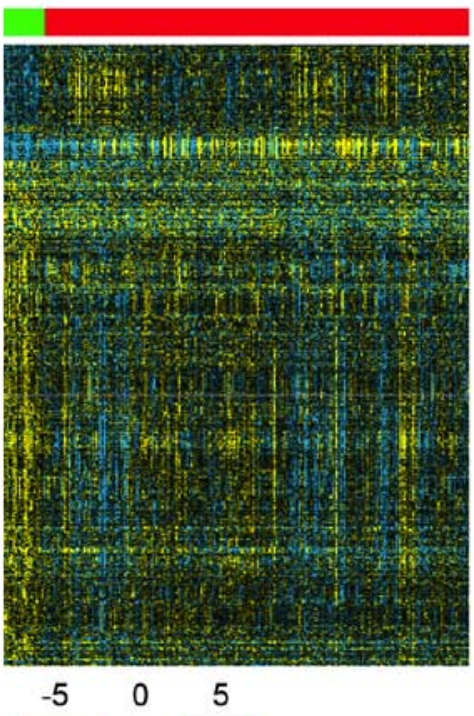

B

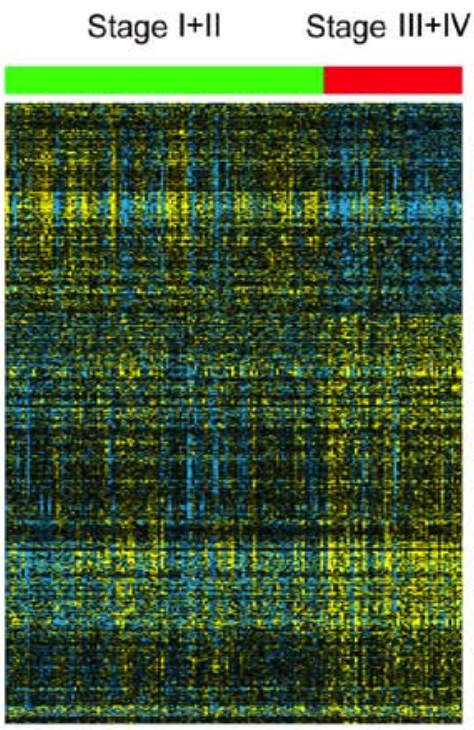

$\begin{array}{lll}-5 & 0 & 5\end{array}$
C Stage I+II Stage III+IV

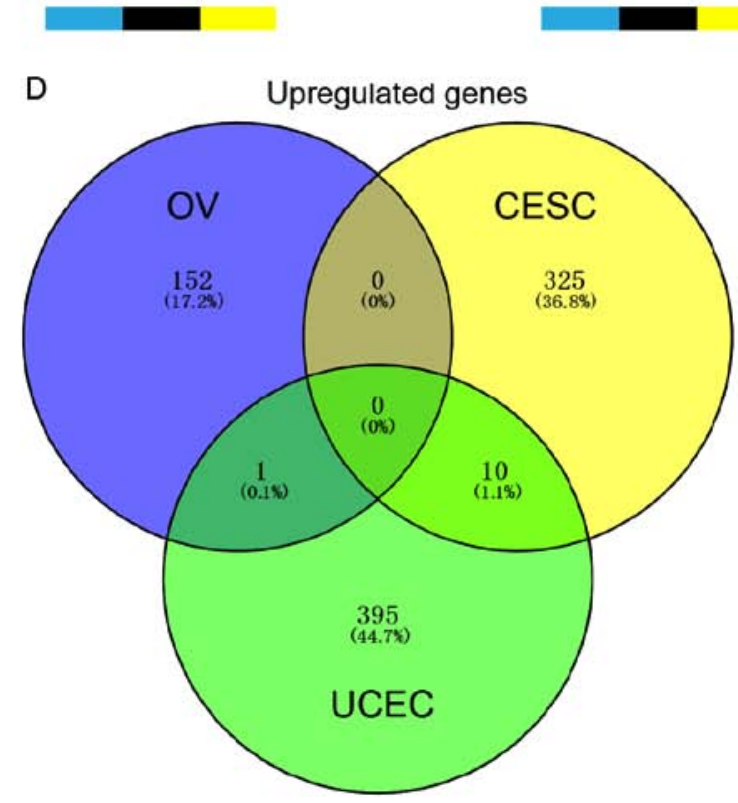

E
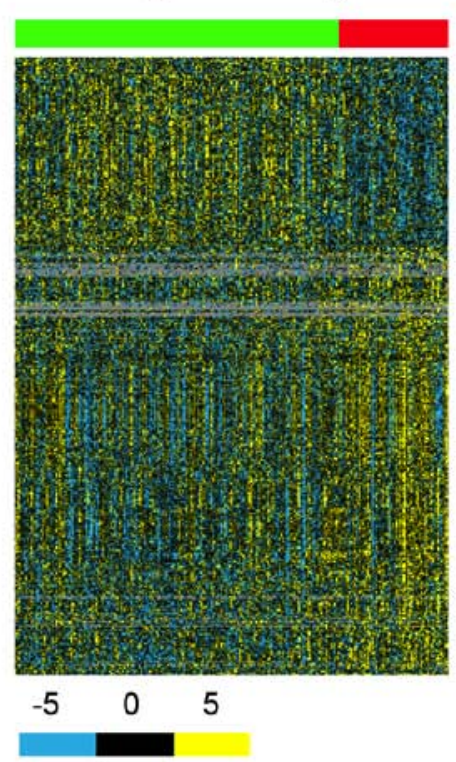

5

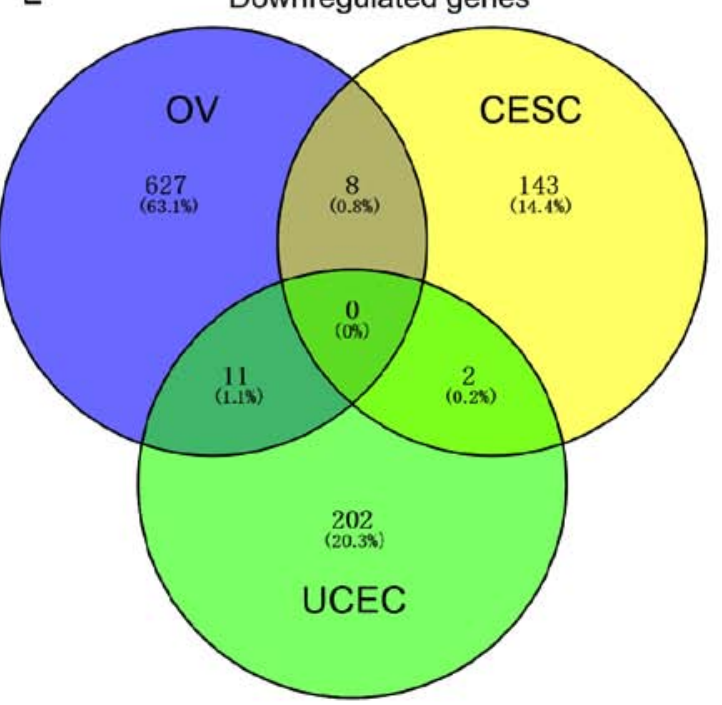

Figure 1. Identification of DEGs in gynecological cancer progression. Hierarchical clustering analysis showing the DEGs $(\mathrm{P}<0.01)$ between $(\mathrm{A})$ Stage I + II and Stage III + IV OV samples, (B) Stage I + II and Stage III + IV UCEC samples, and (C) Stage I + II and Stage III + IV CESC samples. The blue, black and yellow colors refer to 5-, 0 - and -5-folds changes in expression, respectively. Venn diagrams for DEGs whose expression was significantly (D) upregulated and (E) downregulated in OV, UCEC and CESC samples. OV, ovarian serous cystadenocarcinoma; CESC, cervical squamous cell carcinoma and endocervical adenocarcinoma; UCEC, uterine corpus endometrial carcinoma; DEGs, differentially expressed genes.

RBBP7. The CESC progression-associated PPI networks included 69 proteins and 228 edges (Fig. 3C). The top 10 hub genes with the highest degrees involved in CESC progression were identified, including EDN1, GNG10, AGT, EPRS, HSPA4, RIT1, CUL2, GNAII, GPI and GPR68.

Prognostic significance of progression-associated genes in gynecological cancer. Furthermore, Kaplan-Meier curve analysis was conducted to determine the association between progression-associated gene expression and OS in gynecological cancer, using TCGA datasets. The median expression of progression-associated genes was selected as the cut-off to divide the gynecological cancer cases into high- and low-expression groups.
Higher expression of NARS2 and lower expression of TPTI were indicated to be associated with a longer OS time in patients with OV (Fig. 4A and B). Meanwhile, the OS times in SMYD2-high, EGLN1-high, TNFRSF10D-high, FUT11-high, SYTL3-low, MMP8-high and EREG-high expression groups in patients with CESC were significantly shorter compared with their opposing expression groups (Fig. 4C-I). In patients with UCEC, this study indicated that higher expression of SLC5A1, TXN, KDM4B, TXNDC11, $H S D L 2$ and COX16, and lower expression of MGAT4A, DAGLA, ELOVL7, THRB and PCOLCE2 were associated with longer OS times (Fig. 5A-K). These analyses indicated that progression-associated genes could serve as biomarkers for gynecological cancer. 


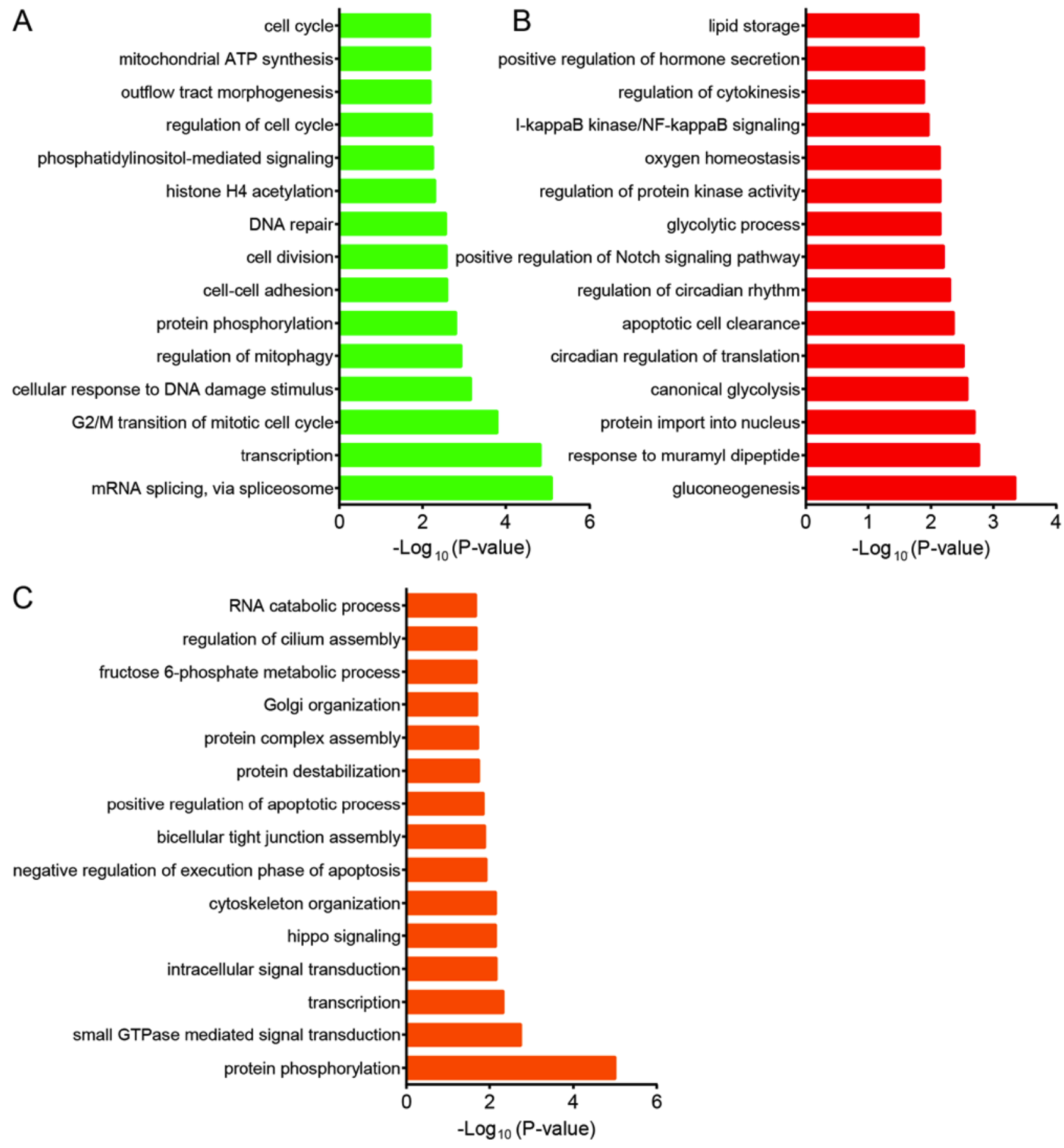

Figure 2. Bioinformatics analysis of DEGs in gynecological cancer progression. Gene Ontology analysis showing DEG-associated biological processes in (A) OV, (B) CESC and (C) UCEC. OV, ovarian serous cystadenocarcinoma; CESC, cervical squamous cell carcinoma and endocervical adenocarcinoma; UCEC, uterine corpus endometrial carcinoma; DEGs, differentially expressed genes.

\section{Discussion}

Gynecological cancer, including OV, CESC and UCEC, are a leading cause of cancer mortality in women (19). In the present study, TCGA datasets were analyzed to identify gynecological cancer progression-associated genes. A total of 153,335 and 406 upregulated, and 646, 153 and 215 downregulated genes were associated with OV, CESC and UCEC progression, respectively. In addition, OV, CESC and UCEC progression-associated PPI networks were constructed to reveal the associations among these genes. Furthermore, Kaplan-Meier curve analysis showed that progression-related genes, such as SMYD2, EGLN1,TNFRSF10D, SLC5A1 and TXN, could serve as prognostic biomarkers for gynecological cancer.

Previous studies have reported certain drivers that are involved in gynecological cancer (20-22). By using TCGA datasets, Berger et al (10) identified various mutated genes in gynecological cancer. CT45 was identified as a chemosensitivity mediator and immunotherapy target in ovarian cancer (20). BRCA1 and BRCA2 are considered to be key regulators of ovarian development and function $(21,22)$. However, the underlying mechanisms regulating cancer progression 
A
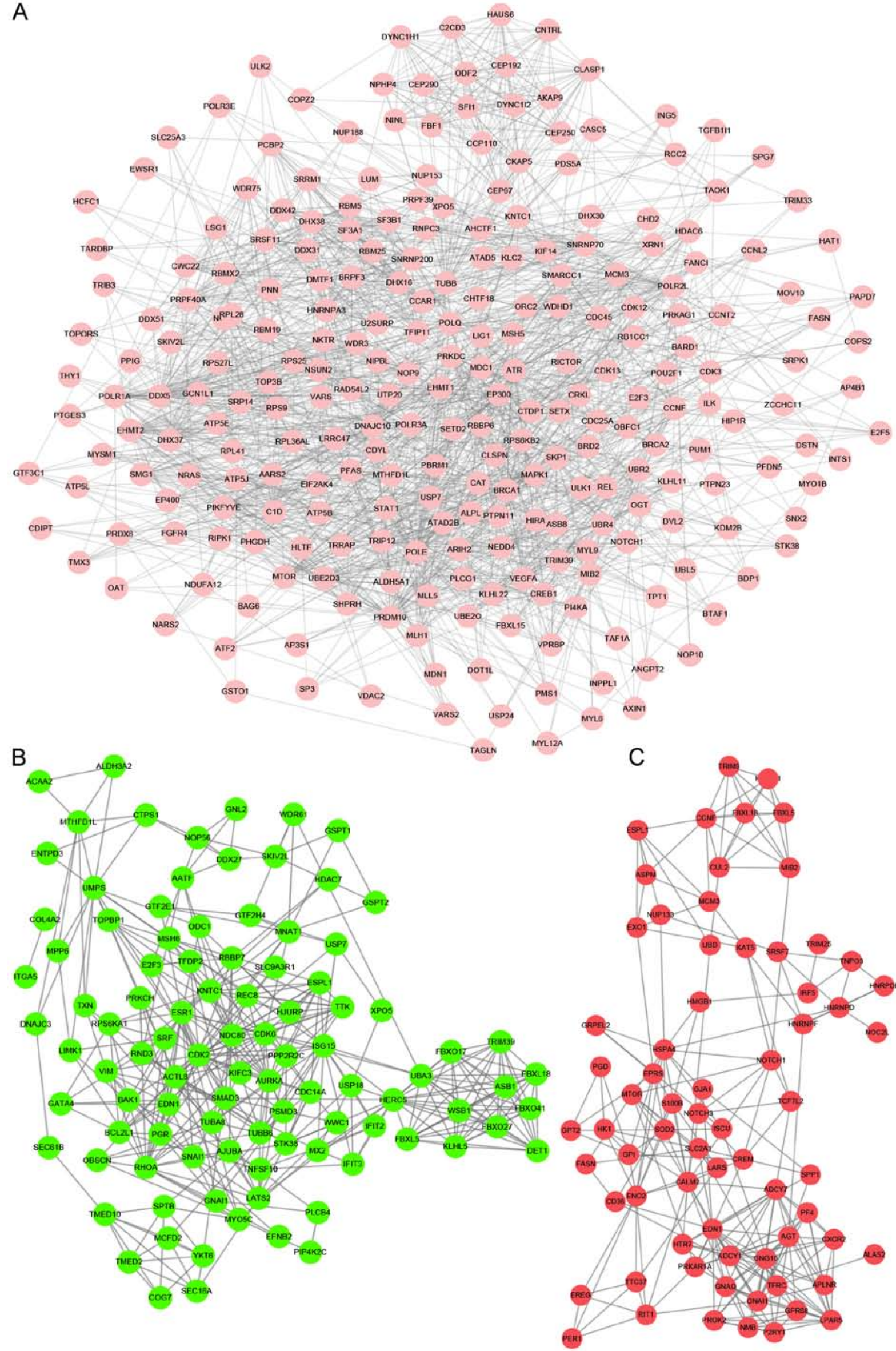

Figure 3. Construction of progression-associated PPI networks in gynecological cancer. Progression-associated PPI networks in (A) OV, (B) UCEC and (C) CESC were constructed. OV, ovarian serous cystadenocarcinoma; CESC, cervical squamous cell carcinoma and endocervical adenocarcinoma; UCEC, uterine corpus endometrial carcinoma; PPI, protein-protein interaction. 

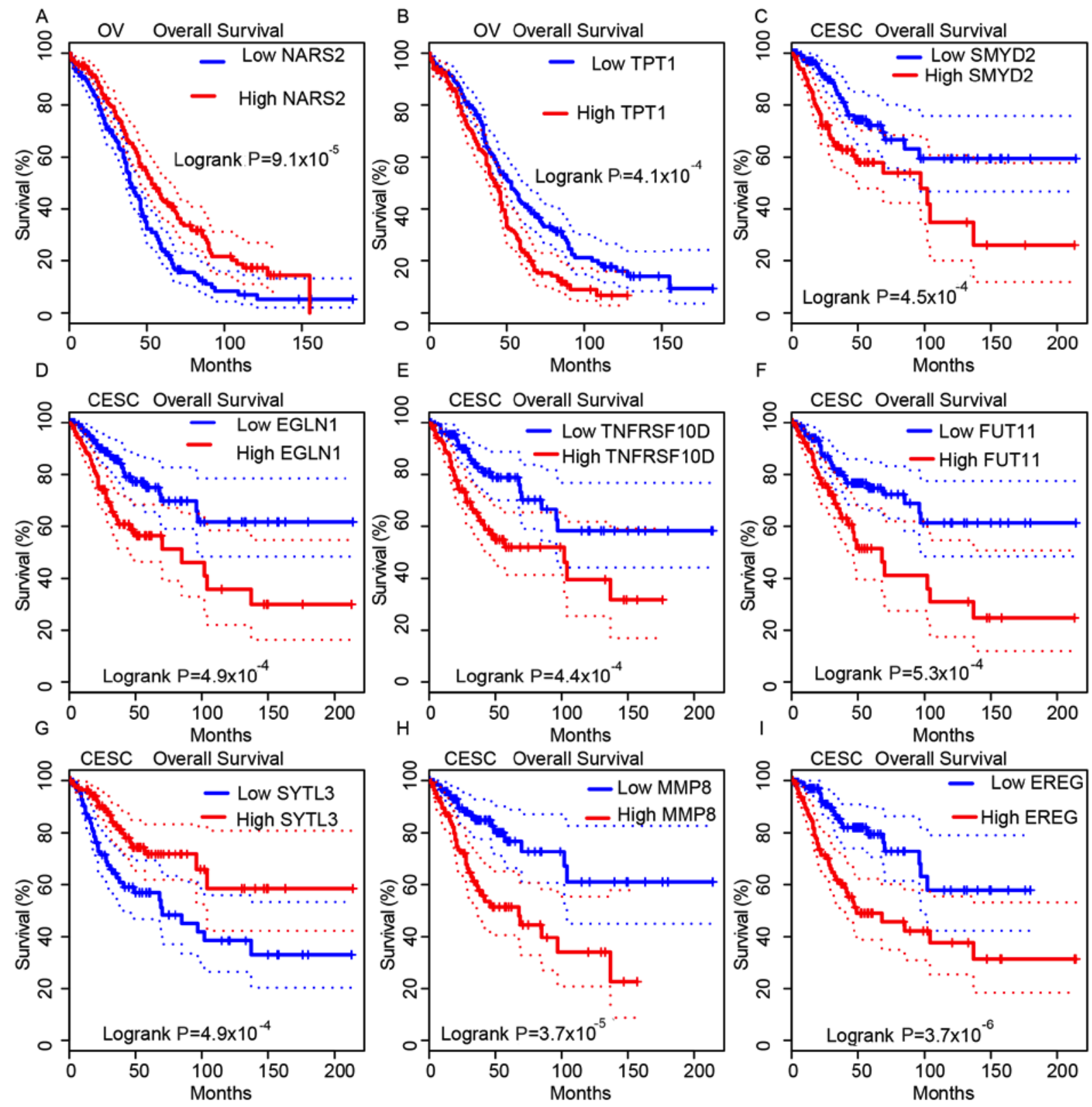

Figure 4. Progression-associated genes are associated with overall survival time in OV and CESC. It was indicated that upregulated expression of (A) NARS2 and lower expression of (B) TPT1 was associated with a longer OS time in patients with OV. The OS times in (C) SMYD2-high, (D) EGLN1-high, (E) TNFRSF10D-high, (F) FUT11-high, (G) SYTL3-low, (H) MMP8-high and (I) EREG-high patients with CESC were significantly shorter. OV, ovarian serous cystadenocarcinoma; CESC, cervical squamous cell carcinoma and endocervical adenocarcinoma.

require further investigation. The present study identified 799 dysregulated genes in OV, 488 dysregulated genes in CESC, and 621 dysregulated genes in UCEC. Only a small number of genes were observed to be dysregulated in more than one gynecological cancer, suggesting that different mechanisms underlie cancer progression in different types of gynecological cancer.

Bioinformatics analyses were also performed, and showed that $\mathrm{OV}$ progression-associated genes were involved in regulating mRNA splicing and cell proliferation-associated BPs. mRNA splicing had been demonstrated to regulate the progression of OV. For example, Snail driving alternative splicing of CD44 by ESRP1 enhances metastasis of OV (23). CESC progression-associated genes were involved in regulating a series of metabolism-related BPs, such as glycolysis and oxygen homeostasis. Glycolysis played a crucial role for the supplication of energy and precursors for human cancer (24). It was also indicated that DEGs in UCEC were associated with protein phosphorylation and small GTPase-mediated signal transduction. In addition, PPI networks were constructed in this study. A few genes were identified to be key regulators in gynecological cancer progression, such as EHMT1 and EHMT2 in OV, EDN1 and GNGIO in CESC, and RHOA and ISGI5 in UCEC.

Over the past decades, efforts have been made to identify accurate biomarkers for gynecological cancer. For instance, upregulation of TRIM44 predicts poor prognosis in epithelial ovarian cancer (25), and LYL1 amplification predicts a shorter survival time of patients with UCEC (26). However, the prognosis of patients with gynecological cancer remains poor. In this study, Kaplan-Meier curve analysis was conducted 
A
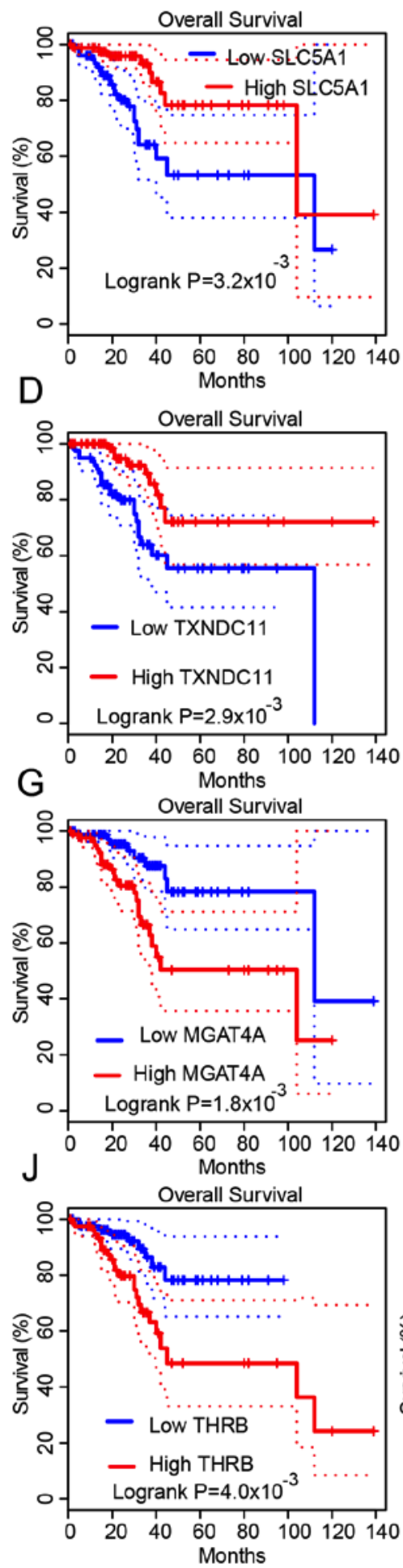

B

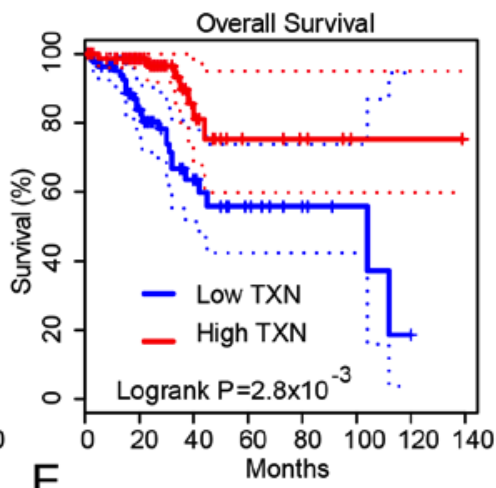

$\mathrm{E}$

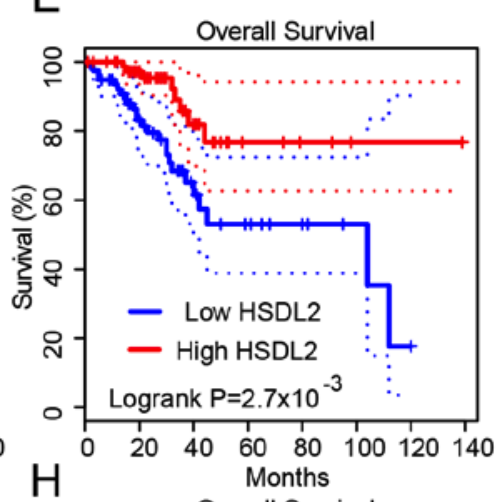

C

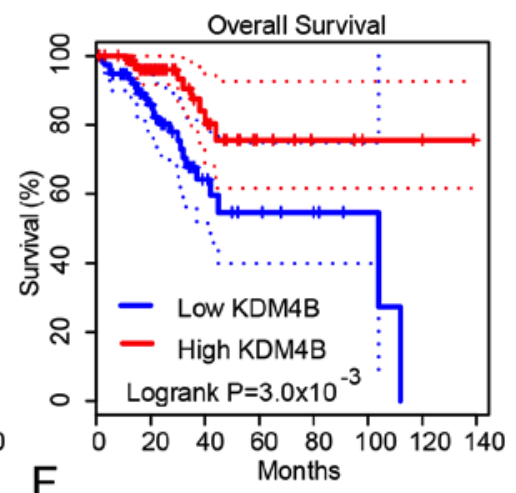

$\mathrm{F}$

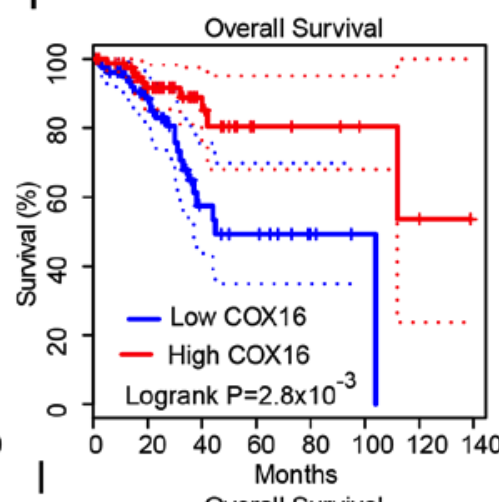

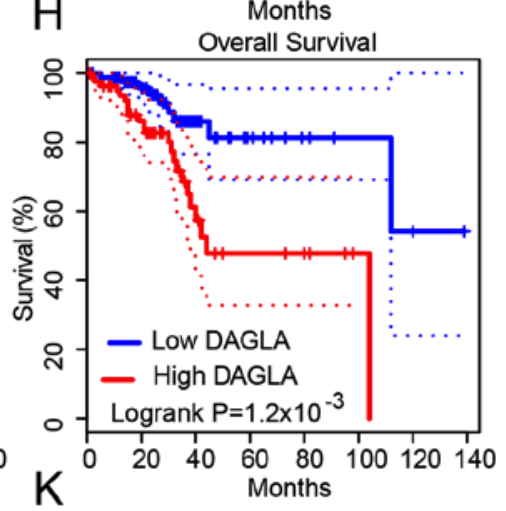
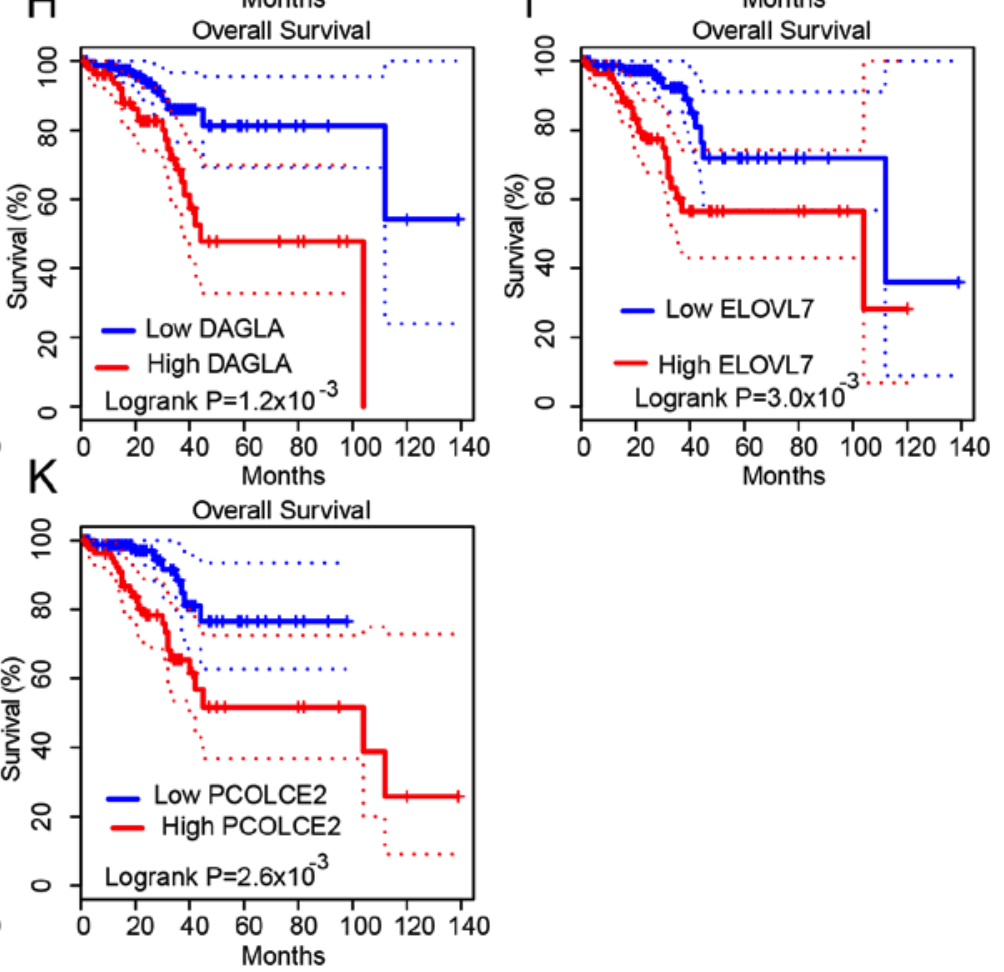

Figure 5. Progression-related genes are associated with overall survival time in UCEC. It was revealed that higher expression levels of (A) SLC5A1, (B) TXN, (C) KDM4B, (D) TXNDC11, (E) HSDL2 and (F) COX16, and lower expression levels of (G) MGAT4A, (H) DAGLA, (I) ELOVL7, (J) THRB and (K) PCOLCE2, were associated with longer OS times in patients with UCEC. UCEC, uterine corpus endometrial carcinoma.

to determine the prognostic value of progression-associated gene expression in gynecological cancer. It was revealed that the dysregulation of NARS2 and TPT1 in OV, the dysregulation of SMYD2, EGLN1, TNFRSF10D, FUT11, SYTL3, MMP8 and $E R E G$ in CESC, and the dysregulation of DC11, HSDL2, COX16, MGAT4A, DAGLA, ELOVL7, THRB and PCOLCE2 in UCSC were associated with OS time. In previous studies, SMYD2 (which encodes SET and MYND domain containing 2 protein) was found to be an oncogene in various types of cancer, including triple negative breast cancer (27), hepatocellular carcinoma (28) and pancreatic cancer (29). FUT11 (fucosyltransferase 11) was identified as a novel prognostic marker for 
clear cell renal cell carcinoma (30). Genetic polymorphisms in $M M P 8$ (encoding matrix metalloproteinase-8) have been reported to be associated with breast cancer (31), bladder cancer (32) and malignant melanoma risk $(33,34)$. HSDL2 (hydroxysteroid dehydrogenase-like 2) serves as an oncogene in ovarian cancer by promoting cell proliferation and cell motility (35). However, the majority of these genes were for the first time reported to be involved in human cancer progression, and these analyses suggested that these progression-associated genes could serve as biomarkers for gynecological cancer.

In the present study, 799 dysregulated genes were identified in OV, 488 dysregulated genes in CESC and 621 dysregulated genes in UCEC. Bioinformatics analysis revealed that mRNA splicing and cell proliferation-associated BPs played important roles in OV progression. In addition, metabolism-related BPs played important roles in CESC progression, and protein phosphorylation and small GTPase-mediated signal transduction played important roles in UCEC progression. OV, CESC and UCEC progression-associated PPI networks were also constructed to reveal the association among these genes. Furthermore, Kaplan-Meier curve analysis showed that progression-related genes were associated with OS time. Finally, NARS2 and TPT1 in OV, SMYD2, EGLN1, TNFRSF10D, FUT11, SYTL3, MMP8 and EREG in CESC, and DC11, HSDL2, COX16, MGAT4A, DAGLA, ELOVL7, $T H R B$ and $P C O L C E 2$ in UCSC were identified as hub genes in cancer progression. Therefore, it is suggested that the present study may assist in the identification of novel mechanisms underlying cancer progression and new biomarkers for gynecological cancer prognosis and therapy.

\section{Acknowledgements}

Not applicable.

\section{Funding}

No funding was received.

\section{Availability of data and material}

The datasets used and/or analyzed during the present study are available from the corresponding author on reasonable request.

\section{Authors' contributions}

$\mathrm{XZ}$ and YW were involved in the conception and design of the research and drafting the manuscript. XZ participated in the acquisition of data, analysis and interpretation of data and statistical analysis. YW participated in the design of the study and performed the statistical analysis. All authors have read and approved the final version of the manuscript.

\section{Ethics approval and consent to participate}

Not applicable.

\section{Patient consent to publication}

Not applicable.

\section{Competing interests}

The authors declare that they have no competing interests.

\section{References}

1. Hutchinson L: Gynecological cancer: True progress in ovarian cancer or just the tip of the iceberg? Nat Rev Clin Oncol 9: 65, 2012.

2. Jha A, Khan Y, Mehdi M, Karim MR, Mehmood Q, Zappa A, Rebholz-Schuhmann D and Sahay R: Towards precision medicine: Discovering novel gynecological cancer biomarkers and pathways using linked data. J Biomed Semantics 8: 40, 2017.

3. Li H, Zhang W, Sun X, Chen J, Li Y, Niu C, Xu B and Zhang Y: Overexpression of kinesin family member $20 \mathrm{~A}$ is associated with unfavorable clinical outcome and tumor progression in epithelial ovarian cancer. Cancer Manag Res 10: 3433-3450, 2018.

4. Wang $S$ and Chen X: Identification of potential biomarkers in cervical cancer with combined public mRNA and miRNA expression microarray data analysis. Oncol Lett 16: 5200-5208, 2018.

5. Krishnan P, Ghosh S, Wang B, Heyns M, Graham K, Mackey JR, Kovalchuk $\mathrm{O}$ and Damaraju S: Profiling of small nucleolar RNAs by next generation sequencing: Potential new players for breast cancer prognosis. PLoS One 11: e0162622, 2016.

6. Yadav SS,LiJ,LaveryHJ,YadavKK and Tewari AK:Next-generation sequencing technology in prostate cancer diagnosis, prognosis, and personalized treatment. Urol Oncol 33: 267 e1-e13, 2015.

7. Weisenberger DJ: Characterizing DNA methylation alterations from the cancer genome atlas. J Clin Invest 124: 17-23, 2014.

8. Cancer Genome Atlas Research Network, Weinstein JN, Collisson EA, Mills GB, Shaw KR, Ozenberger BA, Ellrott K, Shmulevich I, Sander C and Stuart JM: The cancer genome atlas pan-cancer analysis project. Nat Genet 45: 1113-1120, 2013.

9. Sanchez-Vega F, Mina M, Armenia J, Chatila WK, Luna A La KC, Dimitriadoy S, Liu DL, Kantheti HS, Saghafinia S, et al: Oncogenic signaling pathways in the cancer genome atlas. Cell 173: 321-337 e10, 2018

10. Berger AC, Korkut A, Kanchi RS, Hegde AM, Lenoir W, Liu W, Liu Y, Fan H, Shen H, Ravikumar V, et al: A Comprehensive pan-cancer molecular study of gynecologic and breast cancers. Cancer Cell 33: 690-705 e9, 2018.

11. Song J, Ye A, Jiang E, Yin X, Chen Z, Bai G, Zhou Y and Liu J: Reconstruction and analysis of the aberrant IncRNA-miRNA-mRNA network based on competitive endogenous RNA in CESC. J Cell Biochem 119: 6665-6673, 2018.

12. Gao J, Aksoy BA, Dogrusoz U, Dresdner G, Gross B, Sumer SO, Sun Y, Jacobsen A, Sinha R, Larsson E, et al: Integrative analysis of complex cancer genomics and clinical profiles using the cBioPortal. Sci Signal 6: pl1, 2013.

13. Hagemann IS, Cole LL, Cosin JA, Gress DM, Mutch DG and Olawaiye AB: Controversies in gynecologic cancer staging: An AJCC cancer staging manual, eighth edition perspective. AJSP Rev Rep 23: 118-128, 2018.

14. de Hoon MJ, Imoto S, Nolan J and Miyano S: Open source clustering software. Bioinformatics 20: 1453-1454, 2004.

15. Page RD: TreeView. Glasgow University, Glasgow, UK, 2001.

16. Huang DW, Sherman BT, Tan Q, Kir J, Liu D, Bryant D, Guo Y, Stephens R, Baseler MW, Lane HC and Lempicki RA: DAVID Bioinformatics Resources: Expanded annotation database and novel algorithms to better extract biology from large gene lists. Nucleic Acids Res 35 (Web Server Issue): W169-W175, 2007.

17. Ashburner M, Ball CA, Blake JA, Botstein D, Butler $\mathrm{H}$, Cherry JM, Davis AP, Dolinski K, Dwight SS, Eppig JT, et al: Gene ontology: Tool for the unification of biology. The gene ontology consortium. Nat Genet 25: 25-29, 2000.

18. The Gene Ontology Consortium: The gene ontology resource: 20 years and still GOing strong. Nucleic Acids Res 47 (D1): D330-D338, 2019.

19. Abu-Shawer O, Abu-Shawer M, Hirmas N, Alhouri A, Massad A, Alsibai B, Sultan H, Hammo H, Souleiman M, Shebli Y and Al-Hussaini M: Hematologic markers of distant metastases and poor prognosis in gynecological cancers. BMC Cancer 19: 141, 2019.

20. Coscia F, Lengyel E, Duraiswamy J, Ashcroft B, Bassani-Sternberg M, Wierer M, Johnson A, Wroblewski K, Montag A, Yamada SD, et al: Multi-level proteomics identifies CT45 as a chemosensitivity mediator and immunotherapy target in ovarian cancer. Cell 175: 159-170 e16, 2018. 
21. George J, Alsop K, Etemadmoghadam D, Hondow H, Mikeska T, Dobrovic A, deFazio A; Australian Ovarian Cancer Study Group, Smyth GK, Levine DA, et al: Nonequivalent gene expression and copy number alterations in high-grade serous ovarian cancers with BRCA1 and BRCA2 mutations. Clin Cancer Res 19: 3474-3484, 2013.

22. McLaughlin JR, Rosen B, Moody J, Pal T, Fan I, Shaw PA, Risch HA, Sellers TA, Sun P and Narod SA: Long-term ovarian cancer survival associated with mutation in BRCA1 or BRCA2. J Natl Cancer Inst 105: 141-148, 2013.

23. Chen L, Yao Y, Sun L, Zhou J, Miao M, Luo S, Deng G, Li J, Wang J and Tang J: Snail driving alternative splicing of CD44 by ESRP1 enhances invasion and migration in epithelial ovarian cancer. Cell Physiol Biochem 43: 2489-2504, 2017.

24. Pusapati RV, Daemen A, Wilson C, Sandoval W, Gao M, Haley B, Baudy AR, Hatzivassiliou G, Evangelista M and Settleman J: mTORC1-dependent metabolic reprogramming underlies escape from glycolysis addiction in cancer cells. Cancer Cell 29: 548-562, 2016

25. Liu S, Yin H, Ji H, Zhu J and Ma R: Overexpression of TRIM44 is an independent marker for predicting poor prognosis in epithelial ovarian cancer. Exp Ther Med 16: 3034-3040, 2018

26. Kim SI, Lee JW, Lee N, Lee M, Kim HS, Chung HH, Kim JW, Park NH, Song YS and Seo JS: LYL1 gene amplification predicts poor survival of patients with uterine corpus endometrial carcinoma: Analysis of the Cancer genome atlas data. BMC Cancer 18: 494, 2018.

27. Li LX, Zhou JX, Calvet JP, Godwin AK, Jensen RA and Li X: Lysine methyltransferase SMYD2 promotes triple negative breast cancer progression. Cell Death Dis 9: 326, 2018.

28. Zuo SR, Zuo XC, He Y, Fang WJ, Wang CJ, Zou H, Chen P, Huang LF, Huang LH, Xiang H and Liu SK: Positive expression of SMYD2 is associated with poor prognosis in patients with primary hepatocellular carcinoma. J Cancer 9: 321-330, 2018.

29. Reynoird N, Mazur PK, Stellfeld T, Flores NM, Lofgren SM, Carlson SM, Brambilla E, Hainaut P, Kaznowska EB, Arrowsmith $\mathrm{CH}$, et al: Coordination of stress signals by the lysine methyltransferase SMYD2 promotes pancreatic cancer. Genes Dev 30: 772-785, 2016.
30. Zodro E, Jaroszewski M, Ida A, Wrzesiński T, Kwias Z, Bluyssen H and Wesoly J: FUT11 as a potential biomarker of clear cell renal cell carcinoma progression based on metaanalysis of gene expression data. Tumour Biol 35: 2607-2617, 2014.

31. Wang K, Zhou Y, Li G, Wen X, Kou Y, Yu J, He H, Zhao Q, Xue F, Wang J and Zhao X: MMP8 and MMP9 gene polymorphisms were associated with breast cancer risk in a Chinese Han population. Sci Rep 8: 13422, 2018.

32. Wieczorek E, Reszka E, Wasowicz W, Grzegorczyk A, Konecki T, Sosnowski M and Jablonowski Z: MMP7 and MMP8 genetic polymorphisms in bladder cancer patients. Cent European J Urol 66: 405-410, 2014

33. Dębniak T, Jakubowska A, Serrano-Fernández P, Kurzawski G, Cybulski C, Chauhan SR, Laxton RC, Maleszka R, Lubinski J and Ye S: Association of MMP8 gene variation with an increased risk of malignant melanoma. Melanoma Res 21: 464-468, 2011.

34. Palavalli LH, Prickett TD, Wunderlich JR, Wei X, Burrell AS, Porter-Gill P, Davis S, Wang C, Cronin JC, Agrawal NS, et al: Analysis of the matrix metalloproteinase family reveals that MMP8 is often mutated in melanoma. Nat Genet 41: 518-520, 2009.

35. Sun Q, Zhang Y, Su J, Li T and Jiang Y: Role of hydroxysteroid dehydrogenase-like 2 (HSDL2) in human ovarian cancer. Med Sci Monit 24: 3997-4008, 2018.

This work is licensed under a Creative Commons Attribution-NonCommercial-NoDerivatives 4.0 International (CC BY-NC-ND 4.0) License. 